\title{
Laser Ray Tracing versus Hartmann-Shack sensor for measuring optical aberrations in the human eye
}

\author{
Esther Moreno-Barriuso and Rafael Navarro \\ Instituto de Optica "Daza de Valdés," Consejo Superior de Investigaciones Científicas, Serrano 121, \\ 28006 Madrid, Spain
}

Received November 2, 1999; accepted February 7, 2000

\begin{abstract}
A comparison and validation study of Laser Ray Tracing (LRT) and Hartmann-Shack wave-front-sensor (to be referred to as H-S) methods was carried out on both artificial and human eyes. The aim of this work was double. First, we wanted to verify experimentally the equivalence of single- and double-pass measurements for both H-S and LRT. This interest is due to the impossibility of making single-pass measurements in human eyes. In addition, we wanted to validate the LRT technique by comparing it with the H-S wave-front sensor, currently used in many physiological optics laboratories. Comparison of the different methods and configurations carried out in the artificial eye yielded basically the same results in all cases, which means a reciprocal validation of both $\mathrm{LRT}$ and $\mathrm{H}-\mathrm{S}$, in either single- or double-pass configurations. Other aspects, such as robustness against speckle noise or the influence of the size of the entrance $(\mathrm{H}-\mathrm{S})$ or exit (LRT) pupil were studied as well. As a global reference, the point-spread function (PSF) of the artificial eye was recorded directly on a CCD camera and compared with simulated PSF's computed from the experimental aberration data. We also applied these two methods to real eyes (double pass), finding again a close match between the resulting aberration coefficients and also between the standard errors for two normal subjects. However, for one myopic eye with an especially low optical quality (RMS wave-front error $>2 \mu \mathrm{m}$ ) and asymmetric aberrations, the array of spots recorded with the H-S sensor was highly distorted and too difficult to analyze. (C) 2000 Optical Society of America [S0740-3232(00)00506-8]
\end{abstract}

OCIS codes: $330.5370,330.4300$.

\section{INTRODUCTION}

There is an increasing interest in reliable, easy-to-use methods for the measurement of the optical aberrations in the human eye. Rapidly evolving refractive surgery techniques require methods to assess the modifications they induce in the optical quality of the eye. In addition, if we know the wave-front distortion of the eye, it is even possible to correct it, ${ }^{1-3}$ which opens a wide field of new applications such as improving performance on specific visual tasks or retinal surgery.

One of the earliest measurements of the geometrical aberrations of the human eye was carried out by Young ${ }^{4}$ in 1801 with subjective methods. Since then, many authors have measured aberrations in the human eye subjectively (Refs. 5-8 are some classical examples), but objective methods have been developed only more recently. ${ }^{9-13}$ Objective methods have important advantages over subjective ones for practical applications. The latter often depend on the ability of the subject to perform the task (Vernier alignment, discrimination, etc.) and hence on his or her visual (or Vernier) acuity, contrast sensitivity, degree of training and cooperation, attention, etc. These factors limit the application of subjective methods to the foveal or parafoveal region of cooperative subjects. In addition, psychophysical methods tend to be slow, and one has to count on potential mistakes by the subject.

As far as we know, at least five different approaches to the objective measurement of aberrations in the eye have been reported: the Foucault knife-edge test ${ }^{9}$ the objective version of the Howland aberroscope, ${ }^{10}$ phase retrieval from double-pass images, ${ }^{1,3}$ Hartmann-Shack wave-front sensors $^{11,14}$ (to be referred to as $\mathrm{H}-\mathrm{S}$ ), and laser ray tracing $^{12,15}$ (LRT). Although there have been very few comparative studies of these methods, ${ }^{16,17}$ the $\mathrm{H}-\mathrm{S}$ is becoming popular since it offers advantages in terms of robustness, reliability, and rapidity. More important, a large scientific literature and important technological developments are available for its application to the measurement of aberrations that are due to atmospheric turbulence. The H-S method has been compared recently ${ }^{16}$ with Smirnov's psychophysical method. ${ }^{6}$ Nevertheless, psychophysical measurements were so time-consuming that such comparison was limited to a one-dimensional section of the wave aberration, and hence no thorough validation was achieved. A comparative study of the H-S sensor and Howland's aberroscope has recently been reported. ${ }^{17}$

On the other hand, the LRT method has many features in common with the $\mathrm{H}-\mathrm{S}$ method, which permits the design of an experimental procedure in which all parameters (optical path, sampling pattern, etc.) are shared by these two completely objective methods, allowing a rather close and direct comparison for reciprocal validation. With this in mind, the specific goals of the present paper are the following:

1. To validate H-S and LRT separately by direct comparison of double-pass versus single-pass configurations 
in an artificial eye consisting of a high-quality (diffraction-limited) lens plus an interchangeable aberrating plate. In the single-pass case, the flexibility of the LRT method permits us to measure the aberrations either in the object or in the image space for further validation of the basic assumptions. Moreover, a direct recording of the single-pass point spread function (PSF) will provide us with a global reference.

2. Direct comparison of H-S and LRT, both in artificial and in human eyes (double pass) for reciprocal validation. In particular, we want to study the effects of coherence and incoherence of the illumination as well as the effects of entrance and exit pupil size in both cases. In addition, we shall compare different pupil sampling patterns (hexagonal versus square) and densities (0.6- versus 1-mm sampling interval). This last study will be carried out only in LRT, where the sampling pattern and interval are controlled by the computer and hence are very easy to modify.

3. To experience the advantages and drawbacks of both methods in a practical implementation of the measurement of human eyes. Since the two techniques can share the same optical axis as well as most of the components in the setup (optics, mechanics, electronics, software, experimenters and even subjects), we expect that the inherent differences between $\mathrm{H}-\mathrm{S}$ and LRT, as well as their respective pros and cons, will become apparent from this study.

There are two main differences between $\mathrm{H}-\mathrm{S}$ and LRT: $\mathrm{H}-\mathrm{S}$ works in parallel with a fixed sampling pattern and measures the aberrations in the outgoing (second-pass) aerial beam, whereas LRT is sequential with a highly flexible sampling pattern and measures aberrations of the incoming beam (first pass) at the retinal surface. These two important basic differences between LRT and $\mathrm{H}-\mathrm{S}$ imply that they will have different pros and cons, depending on the particular application. Part of this comparative study was already presented at the 1998 Annual Meeting of the Optical Society of America. ${ }^{18}$

As we show below, the results of the comparison have been highly satisfactory, supporting the validity and equivalence of the two techniques.

\section{GENERAL METHODS}

The two methods that are compared in this work, the $\mathrm{H}-\mathrm{S}$ sensor and LRT, are based on the same principle. They measure geometrical aberrations that are the tangents (or partial derivatives) of the wave aberration. Both techniques take a series of samples of the wave front at the exit pupil, although the $\mathrm{H}-\mathrm{S}$ sensor does it in parallel, whereas LRT is sequential in time. In both methods a set of spots is obtained, with each spot corresponding to the $i$ th sample of the beam taken at a given position on the pupil plane with coordinates $\left(\xi_{i}, \eta_{i}\right)$. The first step is to compute the centroid of the $i$ th spot as a maximum-likelihood estimate of its position $\left[X^{\prime}\left(\xi_{i}, \eta_{i}\right)\right.$, $\left.Y^{\prime}\left(\xi_{i}, \eta_{i}\right)\right]$ at the image plane. In an aberration-free optical system, each spot would lie on its reference ideal position, $X^{\prime}=X_{0}^{\prime} ; Y^{\prime}=Y_{0}^{\prime}$. In the presence of aberrations, the spots are displaced from their reference positions; the geometrical aberration is defined simply by the magnitude of these displacements:

$$
\begin{aligned}
\Delta x^{\prime}\left(\xi_{i}, \eta_{i}\right) & =\frac{X^{\prime}\left(\xi_{i}, \eta_{i}\right)-X_{0}^{\prime}\left(\xi_{i}, \eta_{i}\right)}{f^{\prime}} \\
\Delta y^{\prime}\left(\xi_{i}, \eta_{i}\right) & =\frac{Y^{\prime}\left(\xi_{i}, \eta_{i}\right)-Y_{0}^{\prime}\left(\xi_{i}, \eta_{i}\right)}{f^{\prime}} .
\end{aligned}
$$

In this equation the displacements are given in dimensionless tangent units, by dividing by the focal length of the lens used for imaging the spots (CCD objective in Fig. $1)$. This is especially relevant for double-pass measurements in the human eye, where we do not have access to the retinal image plane and thus cannot measure length units but only tangents or angles. For a given point object with coordinates $\left(X_{0}, Y_{0}, Z_{0}\right)$, we can plot the geometrical aberrations $\left(\Delta x_{i}, \Delta y_{i}\right)$ to obtain a spot diagram representing the geometrical aberrations of each sample (pencil of light) of the beam passing through the optical system. The two methods, LRT and H-S, provide the same type of primary data, and hence once we get this set of geometrical aberrations $\left(\Delta x_{i}, \Delta y_{i}\right)$ with either technique, we can apply exactly the same data processing to estimate the wave aberration (see Subsection 2.C). Furthermore, if we can apply the same pupil sampling pattern, then it is possible to compare these two methods directly.

\section{A. Laser Ray Tracing}

The basic principle of the LRT technique has been described before. ${ }^{12,15,19}$ It consists of delivering, sequentially, a bundle of light pencils (nonexpanded laser beams) coming from the same point object $\left(X_{0}, Y_{0}, Z_{0}\right)$ but passing through different locations $\left(\xi_{i}, \eta_{i}\right)$ at the exit pupil plane. In the experiments reported here, the trajectories of the light pencils (rays) are controlled by means of a two-dimensional $X Y$ optical scanner driven by moving-magnet actuators and by additional optics (collimator) when needed. Different sampling patterns (hexagonal, rectangular, polar) and steps can be easily produced. The spot formed by each ray after passing through the artificial or the real eye under study is imaged onto a CCD camera (see Fig. 1). Both the scanner and the camera are controlled and synchronized by means of a personal computer, which is also used to analyze each image, computing the centroid of the spot. Since one of the main goals of this work was to compare the cases of single and double pass, we built different experimental configurations [Figs. 1(a)-1(d)]. One double-pass setup [Fig. 1(d)], dual for H-S and LRT, is employed for both artificial and real eyes. Validation of the double-pass versions of LRT and $\mathrm{H}-\mathrm{S}$ is necessary because double pass is required for making objective measurements in human eyes. There are also two single-pass versions (only for artificial eyes), I and II [Figs. 1(b) and 1(c)], which differ in that object and image spaces are interchanged, and hence the rays propagate in opposite directions. By reciprocity, the spot diagrams should be the same in both versions, and hence these two measurements, I and II, can be used for studying the reliability of the method. Single-pass laser ray tracing had been used before by Sivak and Kreuzer ${ }^{20}$ to study the spherical aberration of the lens in vitro. 


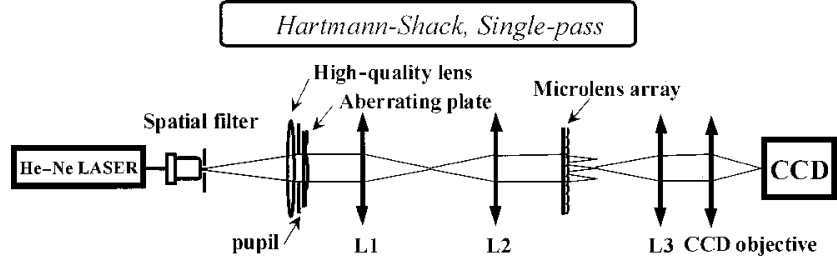

(a)

Laser Ray Tracing, Single-pass II

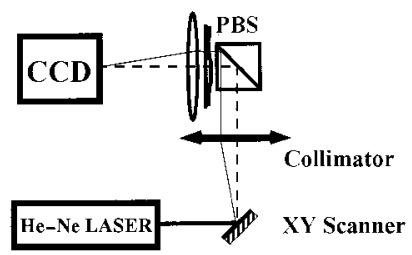

(c)
Laser Ray Tracing, Single-pass I

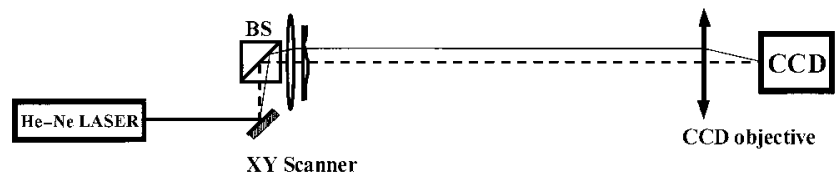

(b)

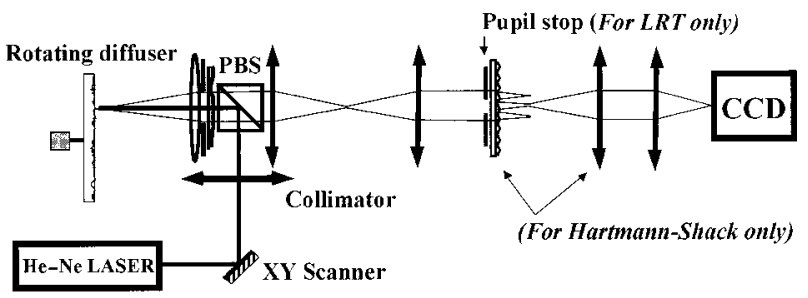

(d)

Fig. 1. Schematic diagrams of the experimental setups. The light source is a TEM 00 polarized red He-Ne laser. The artificial eye is composed of a high-quality lens plus a removable aberrating plate. In all cases the experimental spots are recorded with a cooled digital CCD camera (square pixels, 9- $\mu \mathrm{m}$ size). (a) Single-pass H-S sensor: The pinhole of a spatial filter acts as point object placed at the focal plane of the artificial eye. Lenses L1 and L2 $\left(f^{\prime}=148 \mathrm{~mm}\right)$ project the pupil (1:1 magnification) onto the microlens array. The spots formed by the microlens array are imaged onto the CCD by means of lens L3 $\left(f^{\prime}=200 \mathrm{~mm}\right)$ and the CCD objective $\left(f^{\prime}\right.$ $=105 \mathrm{~mm}$ ). (b) Single-pass LRT (version I): The scanner, placed at the artificial eye's focal plane, delivers a divergent bundle of rays. Each spot is sequentially imaged onto the CCD by the objective $\left(f^{\prime}=55 \mathrm{~mm}\right)$. (c) Single-pass LRT (version II): A set of rays (parallel after passing through the collimator) is delivered onto the artificial eye. Each individual spot is recorded onto the naked CCD (no objective), located at the focal plane. (d) Dual double-pass setup: For LRT the microlens array and L3 are removed. The scanner and the collimator produce a set of parallel rays. Each ray, after being reflected off the rotating diffuser (artificial retina), goes through the eye's pupil and is imaged onto the CCD. The pupil stop, conjugate to the eye's pupil, limits the width of the beam. This stop is removed for H-S measurements, and the scanner is set to zero-deflection angle. The unexpanded beam goes through the center of the artificial eye and is reflected off the rotating diffuser. The second pass (image recording) is the same as in (a).

In the single-pass configurations, the spot formed by each ray (light pencil) is directly recorded by the CCD camera at the image plane [Figs. 1(b) and 1(c)]. However, in version I [Fig. 1(b)] the outgoing beam is collimated (as in the double-pass case) so that the image plane is at infinity, and the CCD objective ( $f^{\prime}$ $=55 \mathrm{~mm}$ ) brings the image plane onto the CCD array. In the double-pass configuration, each light pencil forms a spot on the retina [a rotating diffuser in Fig. 1(d)]. As the entry pupil size is equal to the laser beam size at the pupil plane, $\sim 0.7 \mathrm{~mm}$ (diffraction limited), the first-pass retinal image is a small Gaussian spot (owing to the Gaussian profile of the laser). The light reflected off the (artificial or real) retina passes again through the optics of the eye and forms an aerial image of the retinal spot that is recorded by the CCD camera. This aerial image is the cross correlation between the retinal spot and the PSF of the eye in the second pass. ${ }^{21}$ Thus we have a blurred version of the spot, but its displacement (i.e., centroid location) is the same as that of the single-pass retinal spot $\left(\Delta x_{i}, \Delta y_{i}\right)$ when measured in tangent units [Eq. (1)]. ${ }^{12}$ Nevertheless, we have confirmed this point here by a direct experimental comparison of single- and double-pass measurements.

Although LRT is sequential, it is possible to deliver rays and to record the associated images reasonably fast. Our computer-controlled laser scanner can position the beam and hence deliver rays with frequencies above 1 $\mathrm{kHz}$. Other factors such as the pixel readout of the CCD camera used in the experiments (less than $4 \mathrm{MHz}$ ), exposure time (0.1-0.2 s), and electromechanical shutter of laser and camera, are currently limiting the working frequency to tracing and recording 4-5 rays/s. (At the time we are writing this paper, we have faster electronics and were able to attain frequencies above $20 \mathrm{rays} / \mathrm{s}$ in pilot experiments). One drawback associated with any conventional $X Y$ scanner is that the physical distance between mirrors (11 $\mathrm{mm}$ in our case) induces a slight amount of astigmatism [almost 0.3 diopters (D) for the $200-\mathrm{mm}$ focal-length collimator]. It is easy to eliminate most of it by placing a cylindrical lens in front of the collimator or by using a single mirror on a gimbal mount. Here we have used a $-0.25-\mathrm{D}$ cylinder trial lens (not shown in the figures), and the residual astigmatism $(0.05 \mathrm{D})$ is removed numerically a posteriori by taking a reference measurement with a diffraction-limited lens and applying Eq. (1).

\section{B. Hartmann-Shack Wave-Front Sensor}

The operating principle of the $\mathrm{H}-\mathrm{S}$ wave-front sensor is well known, ${ }^{22,23}$ and its application to the human eye is becoming widespread. ${ }^{1,11,16,24}$ In the $\mathrm{H}-\mathrm{S}$ wave-front sensor, instead of the samples being taken sequentially (as in LRT), a monolithic microlens array located in a plane conjugate to the exit pupil samples the wave front in parallel. Each lenslet selects a light pencil (whose diameter is given by that of the lenslet) and forms a spot of light at its focal plane [see Fig. 1(d)]. For an aberrationfree system, if the object is at the focal point, then the 
emerging wave front is flat, and thus the array of image spots at the microlenses' focal plane has a spatial distribution identical to that of the microlenses themselves (square, hexagonal, etc.). In the presence of aberrations, the light pencils passing through the microlenses are tilted relative to the corresponding aberration-free ones, and thus the image spots are shifted from their ideal or reference position. The tilt is simply the average slope of the wave front across the microlens pupil. ${ }^{23}$ By applying Eq. (1) and subtracting the coordinates of the centroids of the spots obtained for the aberrated system from those obtained with a reference (aberration-free system), we can obtain the geometrical aberrations and the spot diagrams (although some authors prefer to represent the centroids of the spots directly without subtracting the reference). We have built $\mathrm{H}-\mathrm{S}$ sensors working in single[Fig. 1(a)] and double-pass [Fig. 1(d)] configurations. In both cases the $\mathrm{H}-\mathrm{S}$ sensor shares the optical path and the optical axis with the LRT system, as illustrated in Fig. 1(d). In fact, there are only two different setups, one for single pass [Figs. 1(a) and 1(b)] and one for double pass [Fig. $1(\mathrm{~d})$ ]. The LRT single-pass II [Fig. 1(c)] is using the double-pass configuration, the only difference being that the rotating diffuser (artificial retina) is replaced by the CCD array.

In the monolithic microlens array used (manufactured for this purpose at the National Physical Laboratory, Teddington, UK), the lenslets, $f^{\prime}=50 \mathrm{~mm}$, clear aperture $500 \mu \mathrm{m}$, form a hexagonal pattern with a calibrated pitch (center-to-center distance) of $592 \mu \mathrm{m}$. The CCD camera was the same as in LRT but was provided with a $f^{\prime}$ $=105 \mathrm{~mm}$ objective in order to produce a larger magnification.

In all $\mathrm{H}-\mathrm{S}$ measurements the pupil diameter and the sampling pattern were the same. We considered 91 spots (lenslets), sampling a hexagonal pupil area $6.51 \mathrm{~mm}$ (11 lenslets) in diameter. In practice, the microlens array is not perfect (the array itself may have imperfections, and the microlenses may have some prismatic, first-order aberrations), so the spots could shift from the ideal hexagonal pattern even in absence of aberrations. For this reason it is important to record a reference measurement taken on an aberration-free system and to subtract the reference positions from the measured centroids in the aberrated eye, applying Eq. (1). Our reference is the same high-quality lens that we used for the artificial eye of Fig. 1 (without aberrating plate), using only a central low-numerical-aperture-pupil area, which ensures a diffraction-limited performance. The procedure is basically the same as in LRT, permitting the removal of small residual aberrations coming from the experimental measuring system.

The LRT and the H-S systems have been built to be as equivalent as possible to permit the most direct comparison that we could achieve. Hence the scanner was programmed to produce exactly the same sampling pattern as that of the H-S (hexagonal, 91 samples, 592- $\mu$ m step). The only difference is that the microlenses take uniform (circular) samples of $0.5-\mathrm{mm}$ diameter so that the filling factor is less than 1, whereas the laser beam used in LRT is Gaussian with an effective diameter of $\sim 0.7 \mathrm{~mm}$, and thus there is partial overlapping between samples. In addition to this minor difference, the two methods cannot be completely analogous, because (apart from the sequential/parallel nature of LRT and H-S) in LRT the individual samples of the wave front (rays) travel all the way from the object to the image, whereas the $\mathrm{H}-\mathrm{S}$ sensor works with a complete beam, separating the samples at the exit pupil. This difference may have relevant consequences, as explained below. In the single-pass configuration, this feature of LRT permits the interchange of object and image in a simple, natural way [Fig. 1(b) versus Fig. 1(c)]. In the double-pass case, this difference is even more relevant: The $\mathrm{H}-\mathrm{S}$ sensor measures the aberrations of the outgoing beam (second pass), whereas LRT measures the aberrations at the retinal plane (first pass).

\section{Data Analysis}

After application of Eq. (1), the two methods, LRT and $\mathrm{H}-\mathrm{S}$, provide the same type of data, so their analyses are identical. Only the previous computation of the centroids is different. In LRT we record one independent image for each ray. Since each image contains a single spot, it is easy to perform a completely automatic analysis. However, in $\mathrm{H}-\mathrm{S}$ we record a single but larger image that contains the whole array of spots. Thus the image analysis is substantially more complex, since it is necessary to find, index, and analyze each individual spot, which involves pattern recognition and segmentation tasks. In particular, indexing may be difficult for large aberrations, which may severely distort the spot array. Hence we decided to perform spot localization and indexing manually.

The first step (common to both methods) is to subtract a background image, which includes CCD bias correction. For each type of measurement we take background images either by blocking the laser beam (single pass), or by placing a black diffuser in front of the eye (double pass), which also helps to subtract possible reflection artifacts from the image. Then we compute the pixel histogram of the image. A threshold given by $M+3 \sigma$ is set, where $M$ is the mode, as an estimate of the remaining background, and $\sigma$ is the standard deviation. The pixels below threshold are set to zero, and the centroid is then computed. For H-S data, we first localize and index (following a pre-established order) each spot manually. This step is usually easy, but for large and odd aberrations the grid of dots might be severely distorted and the intensity of spots can change strongly, so that indexing may become a difficult task. When all displacements (aberrations) are smaller than the sampling step $(0.592 \mu \mathrm{m})$, the indexing can be easy and automatic: The spots of the recorded pattern are matched sequentially with the ones in the previously registered and indexed reference image. For moderate and low aberrations we verified experimentally that manual and automatic processing yielded the same results. In either case, the centroid is computed within an $11 \times 11$ pixel window around the peak. For both methods we compare the positions of the centroids to the reference, and by applying Eq. (1) we obtain the geometrical aberrations that are the primary data provided by both techniques.

The components $\left(\Delta x_{i}^{\prime}, \Delta y_{i}^{\prime}\right)$ of the ray aberration are the slopes (tangents) of the wave aberration $W(\bar{\xi}, \bar{\eta})$ in 
both axes, where $\bar{\xi}=\xi / R_{p}, \bar{\eta}=\eta / R_{p}$ are dimensionless canonical pupil coordinates and $R_{p}$ is the pupil radius:

$$
\Delta x^{\prime}=\frac{1}{R_{p}} \frac{\partial W(\bar{\xi}, \bar{\eta})}{\partial \bar{\xi}} ; \quad \Delta y^{\prime}=\frac{1}{R_{p}} \frac{\partial W(\bar{\xi}, \bar{\eta})}{\partial \bar{\eta}} .
$$

In this sense we can estimate $W$ by integrating Eq. (2). However, instead of a direct integration that is numerically unstable, it is better to consider an expansion of the wave aberration in terms of Zernike polynomials, ${ }^{22}$ which are well suited to our problem. Here we have considered a seventh-order approximation, that is, 35 terms:

$$
W(\bar{\xi}, \bar{\eta}) \approx \sum_{k=1}^{35} Z_{k} P_{k}(\bar{\xi}, \bar{\eta})
$$

where $Z_{k}$ are the coefficients of the expansion, in micrometers and $P_{k}$ are dimensionless Zernike polynomials (expressions and ordering of polynomials were taken from Malacara $^{22}$ ). Then to estimate $W$ we substitute relation (3) into Eq. (2) to apply the standard method of computing, for each pupil location of our data set, the partial derivatives of the Zernike expansion of $W$. Thus we obtain an overdetermined linear system of equations, where the $Z_{i}$ are the unknowns and which can be solved by standard least-squares fitting. Furthermore, from the estimated $W$, we apply Eq. (2) to compute the derivatives (geometrical aberrations) again so as to compare the resulting "adjusted" spot diagram with the original raw data points in order to verify the goodness of the fitting.

The PSF, $I\left(x^{\prime}, y^{\prime}\right)$, has also been computed in some cases (see Fig. 7 below) as the squared modulus of the Fourier transform of the pupil function ${ }^{25}$ :

$$
I\left(x^{\prime}, y^{\prime}\right)=\left|F T\left(T(\bar{\xi}, \bar{\eta}) \exp \left\{-i\left[\frac{2 \pi}{\lambda} W(\bar{\xi}, \bar{\eta})\right]\right\}\right)\right|^{2},
$$

where $T(\bar{\xi}, \bar{\eta})$ is the transmittance of the pupil, which has been assumed to be uniform for the artificial eye, $W(\bar{\xi}, \bar{\eta})$ is the estimated wave aberration, and $\lambda$ is the wavelength.

\section{ARTIFICIAL EYE: SINGLE AND DOUBLE PASS}

In this section we report experiments made on the artificial eye to validate LRT and H-S. The artificial eye permits us to perform, for each method, a direct comparison of single- versus double-pass measurements. The goal of this study is a direct and objective experimental validation of these methods for application in real eyes in vivo, where we can measure only the light coming out (second pass). We also compare LRT and $\mathrm{H}-\mathrm{S}$ for reciprocal validation. Nevertheless, in Section 4 we repeat this comparison for a definitive validation in human eyes (double pass only). Additional experiments were carried out to assess the influence of several factors, such as coherence and speckle noise, entrance (H-S) and exit (LRT) pupil sizes.

As shown in Fig. 1, the optics of the artificial eye are composed of a low-numerical-aperture diffraction-limited lens $\left(f^{\prime}=250 \mathrm{~mm}\right)$ and an aberrating plate. This plate was made by stretching and twisting a heated microscope glass slide. These deformations induce considerable amounts of low- and high-order aberrations. The plate is mounted on a micrometric translation stage so that it can be displaced laterally outside the optical path and placed back precisely in the same position. The plate was taken out for all preliminary calibrations of the measuring systems and for all reference measurements made with our ideal aberration-free version of the eye. Careful calibrations were made in all configurations by inducing known amounts of defocus and/or astigmatism by using trial lenses as aberrating plates. For double-pass measurements, a white rotating diffuser located at the focal plane of the artificial eye played the role of artificial retina.

\section{A. Experimental Procedure}

Five different aberration measurements were carried out: two for H-S (single and double pass) and three for LRT (single pass, versions I and II, and double pass; see Fig. 1). The two methods share the same optical path as well as most of the optical elements (camera, computer, etc.), so that we can switch them with minimal changes, as illustrated in Fig. 1(d). We checked the correct alignment of both systems carefully by verifying that the chief ray in single-pass LRT (version I) passes through the central lenslet in the microlens array of the $\mathrm{H}-\mathrm{S}$ sensor. The pupil sampling is fixed by the geometry of the microlens array (hexagonal, 91 samples, step $0.592 \mu \mathrm{m}$ ). The artificial eye remains always in the same position to guarantee that aberrations do not change during the study. The light source was a polarized $\mathrm{TEM}_{00}$ red $(633-\mathrm{nm}) \mathrm{He}-\mathrm{Ne}$ laser. The light intensity on the CCD was always kept within the camera's dynamic range by means of neutral density filters. The exposure time was $2 \mathrm{~s}$ for each image in $\mathrm{H}-\mathrm{S}$ and $0.2 \mathrm{~s}$ for each ray in LRT. For each condition four measurements were made, and the mean and standard deviation (SD) were computed. The means are used to compare the different conditions, and the standard deviation is used as an estimate of the experimental variability within a given case, to be compared with the variability among different cases.

The rotation of the diffuser permits us to remove speckle noise in the double-pass measurements. Nevertheless, some additional measurements were made with the diffuser static to study the effect of coherent (speckle) noise on both $\mathrm{H}-\mathrm{S}$ and LRT measurements. In the double-pass case we also studied the effect of the entrance pupil size (for H-S) and of the exit pupil size (for LRT).

For additional validation of the aberration measurements, we performed a direct recording of the single-pass PSF of the artificial eye. For this purpose we modified the setup used for single-pass H-S [Fig. 1(a)] by simply removing the microlens array and lens L3. The PSF is a global reference to be compared with simulated PSF's computed from experimental aberration data (as explained in Subsection 2.C).

\section{B. Preliminary Experiments and Calibrations}

1. Reference Measurements: Aberrations of the Experimental Setups

The first control experiment was to determine the aberrations induced into the measuring systems by the optical 
components, potential misalignments, etc. As we said above, the H-S systems can be affected by imperfections in the microlens array, whereas the two mirrors of the $X Y$ scanner induce astigmatism in LRT. The separation between the two mirrors in the scanner, $d=11 \mathrm{~mm}$ nominal, causes the bundle of rays coming from the object to be affected by astigmatism. ${ }^{19}$ The Sturm interval here is the separation between mirrors, $d$. When a collimator of focal length $f^{\prime}$ is used, the resulting astigmatism in diopters is

$$
D=\frac{d}{\left(f^{\prime}+d / 2\right)\left(f^{\prime}-d / 2\right)} .
$$

In our case, $f^{\prime}=200 \mathrm{~mm}$, the nominal astigmatism is $0.31 \mathrm{D} @ 90^{\circ}$ owing to the $X Y$ orientation of the mirrors. The astigmatism measured experimentally was slightly lower, $0.28 \mathrm{D}$ for single pass, and 0.25 $\mathrm{D}$ for double pass. This astigmatism affects only the fifth Zernike coefficient $\left(Z_{5}\right)$, and we have verified that it can be compensated either before (optically) or after (numerically) the measurements are taken. The optical compensation is carried out by placing a cylindrical lens next to the collimator. We used a cylindrical trial lens, -0.25 D @ 90 ${ }^{\circ}$, and thus the residual astigmatism was always less than $0.05 \mathrm{D}$. (This astigmatism, as other residual aberrations in the system, are compensated numerically a posteriori simply by application of Eq. (1), where $X_{0}^{\prime}, Y_{0}^{\prime}$ represent these residual aberrations obtained in the reference measurements). Apart from controlling the scanner's astigmatism, reference measurements helped to fine tune the focus and alignment of all the optical elements in the system. Although most coefficients were nonzero even in the reference measurements (especially for $\mathrm{H}-\mathrm{S}$ ), the RMS wavefront error was below $\lambda / 5$ in all reference measurements.

\section{Calibrations with Trial Lenses}

After the reference measurements, we used trial lenses (sphere, cylinder, or both) of known refractive power as aberrating plates. In this way we produced controlled and known amounts of second-order aberrations (defocus and/or astigmatism). Measurements obtained in eight cases (powers of trial lenses ranging from -1 to $+1 \mathrm{D}$ ) resulted in an average difference between measured and nominal values for defocus and astigmatism (estimated from the Zernike coefficients) of $5 \%$.

\section{Coherent and Incoherent Measurements}

It has been pointed out ${ }^{26}$ that speckle noise can be a serious drawback in $\mathrm{H}-\mathrm{S}$ measurements in the human eye. We wanted to study its effect in both $\mathrm{H}-\mathrm{S}$ and LRT methods. Speckle noise appears when a coherent beam is scattered by a diffuser. In the human eye there is a diffuse reflection at the retina, and thus coherent noise appears in the second-pass aerial image. Eye movements help to blur speckle and break the coherence of the beam for long-enough exposure times. However, for short exposures, speckle noise has a high contrast ${ }^{27,28}$ and can strongly affect the measurements. We simulated long exposures in the artificial eye by using a rotating diffuser as artificial retina, and we measured the aberrations of the artificial eye by means of the double-pass $\mathrm{H}-\mathrm{S}$ and LRT, both in the incoherent case (rotating diffuser) and in the coherent one (diffuser static). The results obtained are illustrated in Fig. 2. For the H-S, all the spots are contained within one image (upper panel), whereas only one spot is stored in each LRT image (lower panels). In the latter case the spot corresponds to a marginal ray in the coherent (left) and incoherent (right) cases. For LRT the spots are much larger than the speckle grains, and hence the position of the centroid is not biased significantly by speckle noise. The relative RMS difference between coherent and incoherent wave-aberration data was as low as $1.4 \%$. This result suggests a high robustness against coherent noise. However, in the $\mathrm{H}-\mathrm{S}$ image the spots appear smooth and distinct in the incoherent image, but in the coherent case the speckle grains have a size comparable to that of the spots, which are heavily distorted. Not only can this cause important biases in the position of the centroid, but some spots appear mixed up and are even difficult to recognize. In fact we had to desist from analyzing this image.

\section{Effect of Pupil Size}

In double-pass measurements the light crosses the optics of the eye twice. Typically, one pass is used for measurement and the other (second pass in LRT and first pass in $\mathrm{H}-\mathrm{S}$ ) has an auxiliary role. The goal here is to study the effect of this auxiliary pass. In the incoherent case the spots that we record are the cross correlation between the PSF's of both passages. ${ }^{21}$ In LRT this is the cross correlation between the Gaussian spot formed by the laser pencil on the retina ${ }^{12,15}$ and the eye's PSF in the second (auxiliary) pass. Conversely, in $\mathrm{H}-\mathrm{S}$ each spot is the cross correlation between the eye's PSF (first, auxiliary pass) and that of a single microlens (second pass). In both cases the size and shape of the spot, and hence the accuracy in determining its location, depends on the eye's PSF, which depends strongly, in turn, on the diameter of

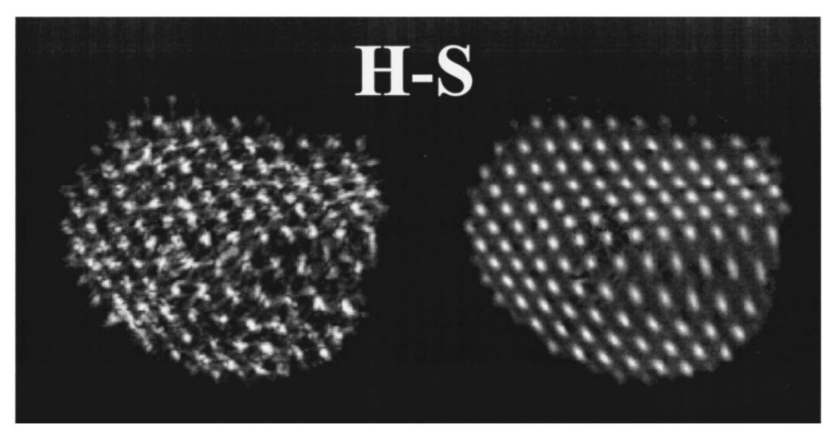

\section{Coherent Incoherent}

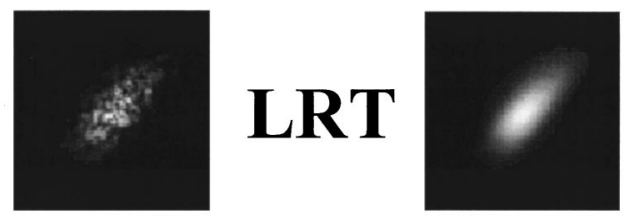

Fig. 2. Effect of speckle noise in double-pass recordings: left column, coherent recordings; right column, incoherent recordings. Upper panels, H-S; lower panels, LRT. 


\section{Effect of entrance pupil diameter}
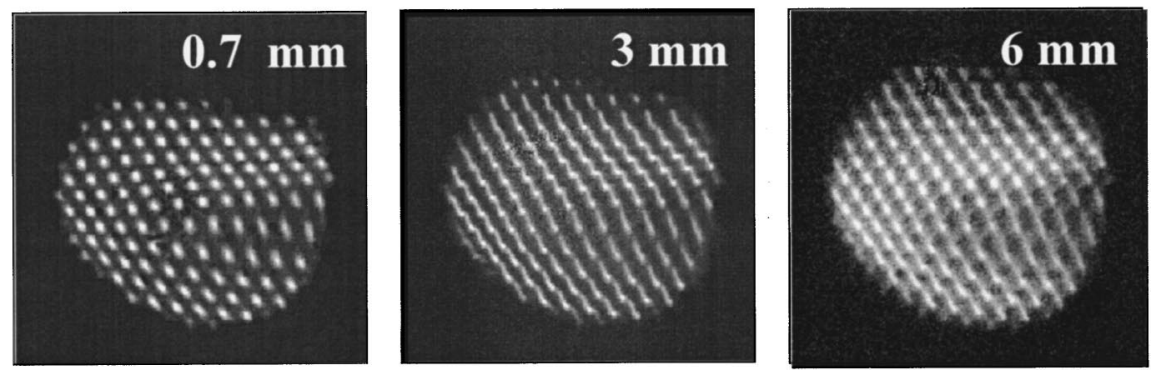

Fig. 3. Double-pass H-S recordings in the artificial eye, obtained with different entrance pupil diameters of $0.7 \mathrm{~mm}, 3 \mathrm{~mm}$, and $6 \mathrm{~mm}$. Blur increases with pupil size.

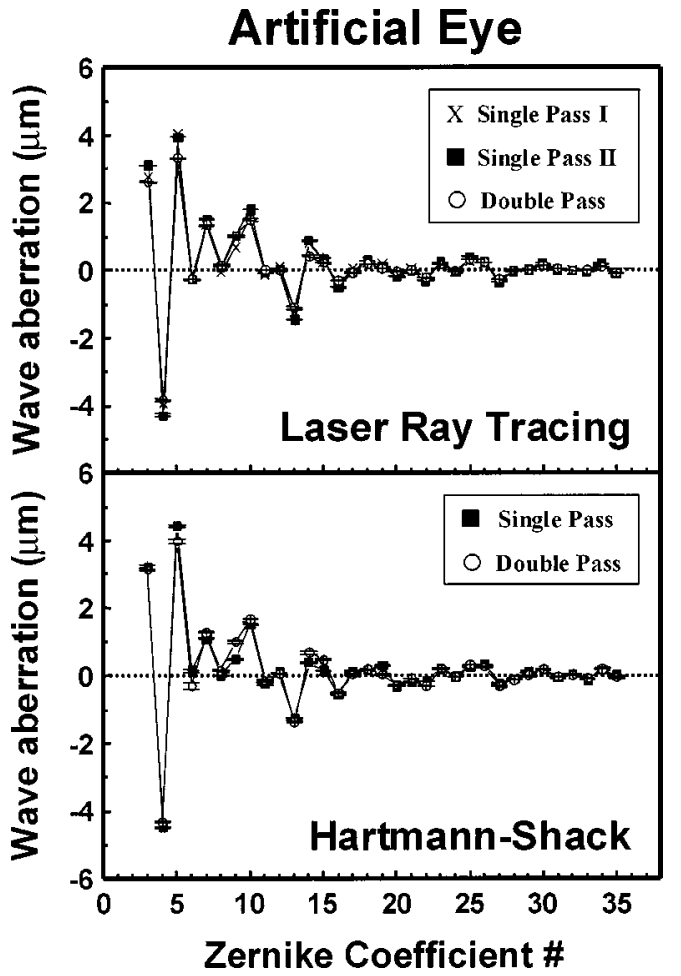

Fig. 4. Zernike coefficients (in micrometers) of the artificial eye obtained with five different conditions. Error bars represent the variability (standard error) obtained in four measurements.

the auxiliary beam (entrance pupil in $\mathrm{H}-\mathrm{S}$ and exit pupil in LRT). We studied this effect in the following control experiments.

We compared three different double-pass $\mathrm{H}-\mathrm{S}$ measurements, modifying the entrance pupil diameter. First we used the unexpanded beam, which corresponds roughly to a $0.7-\mathrm{mm}$ pupil diameter [the case shown in Fig. 1(d)]. For the other two measurements we used entrance pupils of 3 and $6 \mathrm{~mm}$ for which the beam had to be expanded. Figure 3 compares the three resulting $\mathrm{H}-\mathrm{S}$ recordings obtained, from left to right, for 0.7-, 3-, and 6 -mm pupils. It is clear that the location of the spots does not change even though blur increases with pupil size. For a 3-mm pupil, astigmatism of the artificial eye produces an elongation of the spots so that they are somewhat mixed along the diagonal, which causes some extra difficulty in computing the centroids. For the 6-mm pu- pil, the blur is substantially higher, affecting the contrast and making it even harder to estimate the centroids. As a result, the relative RMS difference in the wave-front distortion measured with 0.7 - and 6 -mm pupils was $17 \%$. We attribute this $17 \%$ to the additional error that is due to the inaccuracy in determining the centroids for the largest entrance pupils. Consequently, we decided to use the smallest, 0.7-mm pupil size in all subsequent measurements with this artificial eye.

For LRT there was an equivalent effect when we changed the size of the exit pupil. The spots changed in the same fashion as in H-S. However, since the different spots are recorded sequentially in different images, they do not interact, and hence we can tolerate larger aberrations, that is, larger exit pupils. We recorded two measurements with exit pupils of 3 and $6 \mathrm{~mm}$ diameters. The relative RMS difference was $\sim 4 \%$. This difference is not very high but is significant. For subsequent measurements we again chose the smallest pupil, of 3-mm diameter [see Fig. 1(d)]. In this case we did not try smaller pupils, because it is important to use as much of the energy coming from the eye as possible. A 3-mm pupil represents a good compromise; we had a reasonably good localization of the centroids of spots while at the same time we did not throw away too much light, which is especially important in measuring human eyes.

\section{Hartmann-Shack versus Laser Ray Tracing and} Single versus Double Pass

After these control measurements, we carried out the main experiment, which is the measurement of the aberrations of the artificial eye in five different ways. Figure 4 compares the results obtained in single-pass (conditions I and II for LRT) and double-pass measurements, for both LRT and H-S. The figure displays the sets of Zernike coefficients, in micrometers, obtained in each case. The error bars represent the standard deviation for each condition. These error bars are small in all cases, which means a low experimental variability and high reproducibility of measurements with the artificial eye. Tilt terms $\left(Z_{1}\right.$ and $\left.Z_{2}\right)$ have not been included, because their values for single- and double-pass measurements are not directly comparable. There is a remarkable agreement between single- and double-pass measurements. Several coefficients show differences larger than their corresponding error bars, but they are always reasonably low. We can observe the same basic trend in both LRT and H-S 
cases. As expected, no coefficient associated with the double-pass measuring system seems to be systematically biased. Also expected, there is no partial cancellation of asymmetrical aberrations because of the double pass of the light through the optics of the eye. In addition, versions I and II of single-pass LRT measurements provide almost identical results, which is an additional validation of the reversibility and equivalence of the incoming and outgoing beams in the double pass. Nevertheless, it is important to note that this comparison was made with incoherent recordings and with limiting of the width of the auxiliary beam to control blur. These two factors can have a strong influence on double-pass measurements and need to be controlled, especially in H-S measurements.

If we compare the values of the coefficients in the two plots of Fig. 4, they also look similar. This similarity is even more patent in Fig. 5, which compares four different estimates of the wave aberration, computed by applying Eq. (3) to the different data sets of Fig. 4 (only one version of single-pass LRT is plotted since the two versions look the same). The contour step is $0.5 \mu \mathrm{m}$ of wave-front distortion. In addition to tilt coefficients, defocus $\left(Z_{4}\right)$ was

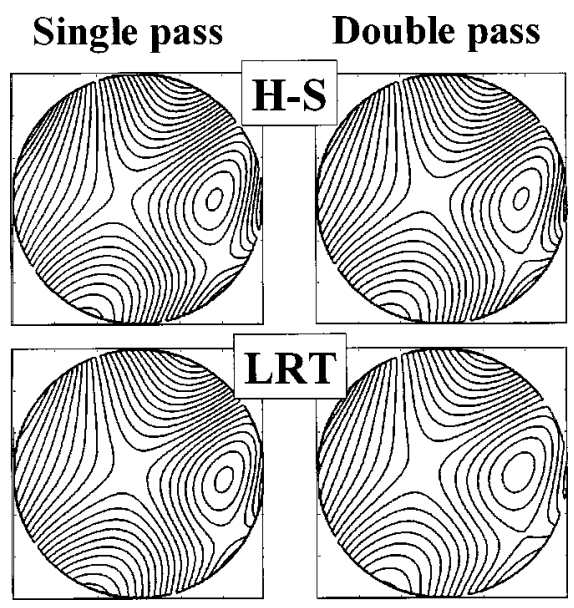

Fig. 5. Contour plots of the wave aberration obtained for $\mathrm{H}-\mathrm{S}$ and LRT in single- and double-pass configurations (the singlepass LRT plot corresponds to version II). The step between adjacent contour lines is $0.5 \mu \mathrm{m}$. Tilts and defocus $\left(Z_{4}\right)$ have not been included, to enhance the asymmetrical and higher-order features of the wave aberration.

\section{Global Average of 4 methods}

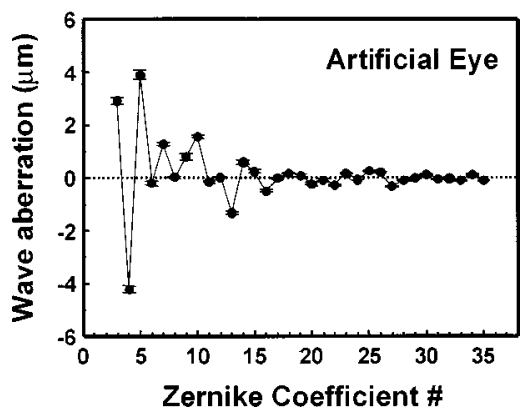

Fig. 6. Average Zernike coefficients (in micrometers) of the artificial eye. Symbols represent the average of the five types of measurements, and error bars represent the variability (standard error) among all conditions. set to zero to represent the case of best focusing and to enhance asymmetric features. The four plots are very similar. In particular, the two single-pass measurements look almost identical, whereas one can tell some slight differences between the double-pass plots. The estimates of the RMS wave-front error are $2.15 \mu \mathrm{m}$ (LRT single-pass version I), $2.28 \mu \mathrm{m}$ (LRT single pass, version II), $1.91 \mu \mathrm{m}$ (LRT double pass), $2.37 \mu \mathrm{m}$ (H-S single pass) and $2.26 \mu \mathrm{m}$ (H-S double pass). The largest difference appears between the two opposite cases, H-S single pass and LRT double pass, but the agreement is quite reasonable. An important part of the mismatch can be attributed not to real systematic differences inherent in the different methods but to variability in experimental conditions. Although we tried to be especially careful in aligning and focusing optical components, we were using as many as five different versions of the system (plus another one for PSF recording), and it is very difficult to reproduce exactly the same conditions in all cases. Figure 6 demonstrates this fact. It represents the same data as in Fig. 4 but in a different way. Now the data points represent the global average of these four different results, and the error bars show the standard deviation among the four conditions. These error bars have a size similar to but slightly larger than those in Fig. 4, which represented experimental variability within each condition. Thus we obtain basically the same result in the four conditions that we are comparing. Variability between conditions is slightly larger than variability within the same condition simply because we are modifying the setup or even using a different setup.

Apart from this comparison between the different conditions, we recorded the single-pass PSF directly as a general reference for all cases. Figure 7 compares this direct recording of the PSF with those computed (simulated) for the four different types of measurements. The size of the PSF is approximately $1 \mathrm{deg}$ of field. The strong resemblance between the reference (direct recording) and the four simulated PSF's is a further global validation of both methods, H-S and LRT, working in single- or double-pass configurations.

\section{HUMAN EYES}

In Section 3 we presented results obtained with artificial eyes, where all experimental conditions can be easily controlled. Measurements in living eyes present additional problems (eye and head movements and alignment, low reflectance of the retina, subjects' comfort and safety, etc.), and hence a complete comparison and validation must include as well real measurements in human eyes.

\section{A. Experimental Procedure}

Three subjects participated in this study, two females (EM and SM, ages 25 and 28) and one male (RN, age 41). For all of them the right eye was measured. Their refractive spherical errors ranged from $-5.6 \mathrm{D}(\mathrm{SM})$ to $-0.5 \mathrm{D}$ (EM). Subject SM wore her contact lenses during the experiment. Measurements were made under cycloplegia, achieved by instillation of one drop of cyclopentolate $1 \%$, administered $30 \mathrm{~min}$ before starting the measurements 


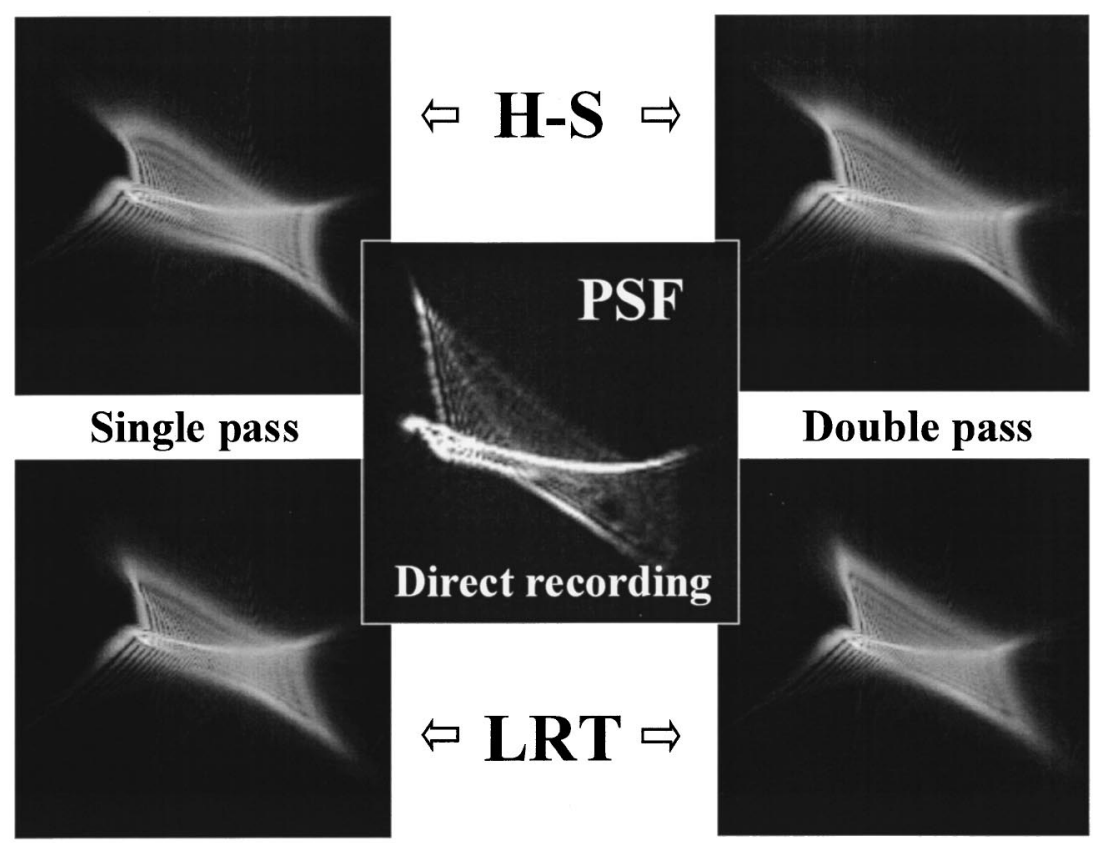

Fig. 7. PSF of the artificial eye recorded directly on the CCD (center) compared with simulations computed from aberration data: H-S (upper panels), LRT (lower panels), in single- (left panels) and double-pass (right panels) configurations. The horizontal size of the PSF is approximately $1^{\circ}$ of field.

and a second drop 5 min after the first one. One additional drop was given after every hour to ensure continuous complete cycloplegia.

The setup [see Fig. 1(d)] was modified for human eye measurements. Instead of the artificial eye, we placed a bite bar mounted onto an $X Y Z$ micropositioner for head fixation. An additional head rest ensured a steady head position throughout the experiment. For pupil alignment $(X, Y)$ and focusing $(Z)$ the eye was illuminated with a set of six infrared LED's. The image, recorded by an infrared camera, was displayed on a TV monitor and centered with respect to a reticle overlay previously aligned with the optical axis of the system. A pellicle beam splitter placed between lenses L1 and L2 [in Fig. 1(d)] was used to monitor the pupil and also to insert a fixation test (by means of another beam splitter). This test, conjugate with the point object, consisted of a radial grating with a well-defined center that had maximum frequency content. This grating was back illuminated with red light, whereas the measurement wavelength was now $543 \mathrm{~nm}$ from a green $\mathrm{He}-\mathrm{Ne}$ laser. In this way, it was easy for the subject to discriminate fixation (red) from the laser probe (green). We changed the working wavelength because $543 \mathrm{~nm}$ is close to the peak of the spectral sensitivity $\left(V_{\lambda}\right)$ of the eye. The laser power entering the eye was always (for both $\mathrm{H}-\mathrm{S}$ and LRT) below $10 \mu \mathrm{W}$, which is $\sim 3$ log units below safety standards for the exposure times used. $^{29}$ An electromechanical shutter, synchronized with both the camera and the scanner, was used for illuminating the eye only during image registration and recording. Apart from these additional elements, we changed lenses L1 and L2, now having $f^{\prime}=250 \mathrm{~mm}$ instead of $148 \mathrm{~mm}$, for convenience.

For LRT measurements the exposure time was $200 \mathrm{~ms}$ per ray, and we applied a binning factor of 2 to the recorded $128 \times 128$-pixel subframes to work with a 64 $\times$ 64-pixel image format. For $\mathrm{H}-\mathrm{S}$ the exposure time was typically $2 \mathrm{~s}$, no binning was applied, and the images had $256 \times 256$ pixels. The field angle subtended by one pixel (before binning) was 1.13 arc min for LRT and 0.59 arc min for H-S. Each measurement was made four times, and the mean and standard deviation of the results were computed the same way as before.

\section{B. Results: Hartmann-Shack versus Laser Ray Tracing}

Figure 8 displays the wave aberration obtained with $\mathrm{H}-\mathrm{S}$ and LRT for subjects EM and RN. Tilts are not included. In addition, for $\mathrm{RN}$ the defocus coefficient $\left(Z_{4}\right.$ $\approx-4 \mu \mathrm{m}$ ) was much higher than the other coefficients and thus was not considered in these plots, in order to enhance other aberrations. We can appreciate that the agreement between $\mathrm{H}-\mathrm{S}$ and LRT results is reasonable for both subjects. As expected, differences between $\mathrm{H}-\mathrm{S}$ and LRT are greater in human eyes than in the artificial eye, but experimental variability was greater too: Error bars in the Zernike coefficients were approximately four times higher in both methods. Average error-bar values for $\mathrm{H}-\mathrm{S}$ were $0.08 \mu \mathrm{m}$ for EM, $0.065 \mu \mathrm{m}$ for $\mathrm{RN}$, and 0.02 for the artificial eye; average error-bar values for LRT were $0.05 \mu \mathrm{m}$ for EM, $0.04 \mu \mathrm{m}$ for $\mathrm{RN}$, and 0.012 for the artificial eye. This higher experimental error can be explained by head and eye movements and to some extent by the low reflectivity of the retina, which produces a decrease in the signal-to-noise ratio and contrast of the spots. Results for the third subject, SM, are not included because we could not satisfactorily process the H-S image, which is shown in Fig. 9. In this myopic eye (-5.6 D), in addition, the RMS wave-front error estimated by LRT was significantly larger than in the other subjects. In particular, the contribution of 3rd-order asymmetrical aberrations was much higher. Several spots in Fig. 9, 
mainly in the upper central part, present a too-low intensity and contrast and could not be analyzed properly. Although it was possible to localize most of the spots visually, the error in estimating the centroid was too large,

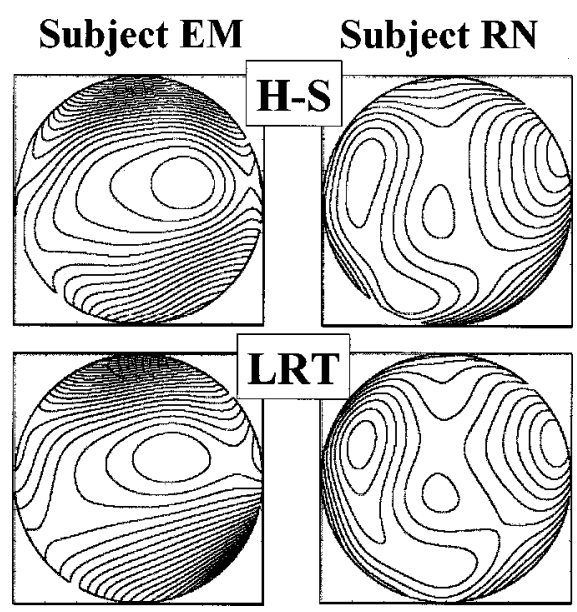

Fig. 8. Contour plots of the wave aberration for subjects EM and RN, obtained with $\mathrm{H}-\mathrm{S}$ and LRT. Tilt terms were not considered, and for RN neither was defocus $\left(Z_{4}\right)$. The step between adjacent contour lines is $0.5 \mu \mathrm{m}$.

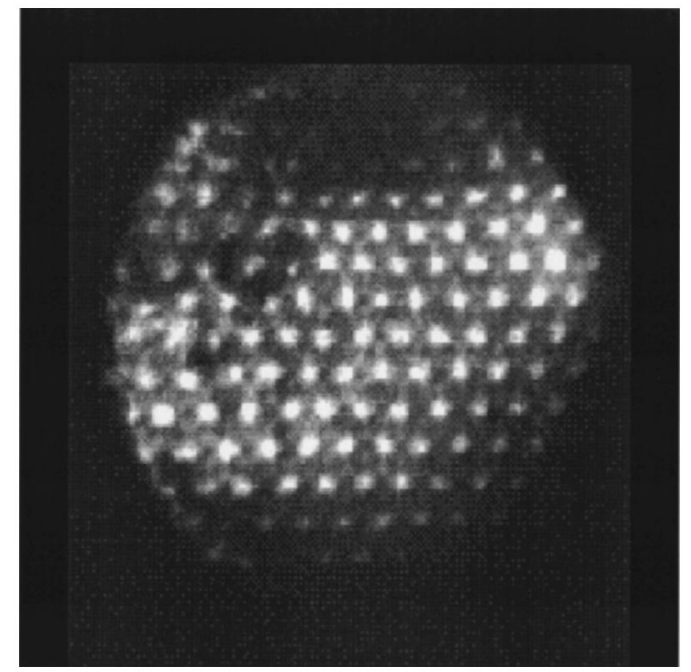

Fig. 9. H-S recording for subject SM, who presents low optical quality and strong asymmetrical aberrations.

Subject RN

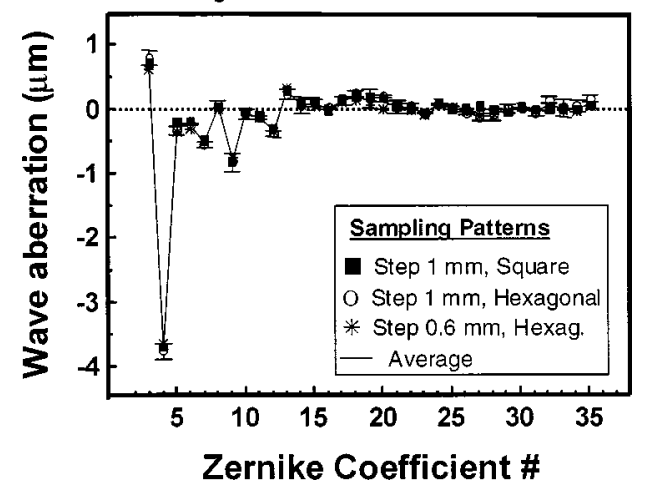

Fig. 10. Invariance against changes in the pupil sampling pattern for subject RN. The Zernike coefficients obtained with LRT and different sampling patterns (square and hexagonal) and step sizes $(0.6$ and $1 \mathrm{~mm})$, show basically the same values. and the resulting $\mathrm{H}-\mathrm{S}$ Zernike coefficients were not consistent and differed too much from LRT data.

These results evidence a partial validation of the methods. The method has been validated for healthy normal eyes, where aberrations are not too high (RMS wave-front error $1.25 \mu \mathrm{m}$ for EM and $0.51 \mu \mathrm{m}$-defocus not included-for RN). However, for a myopic subject with larger aberrations (2.1- $\mu \mathrm{m}$ RMS), which constitute a clinically interesting case, we could not obtain a reliable measurement with $\mathrm{H}-\mathrm{S}$, and hence we could not validate the $\mathrm{H}-\mathrm{S}$ method. In LRT each spot is recorded and processed independently, which makes it notably more robust against noise and, more important, against large changes in the magnitude of the input (aberrations).

\section{Effect of Sampling Pattern}

We performed a final control experiment to study the influence of pupil sampling pattern and frequency. This has an important practical interest because one typically wants to optimize a method by using the simplest configuration that guarantees proper and reliable functioning. Until now, we were using a truncated Zernike polynomial expansion with 35 terms but were recording 91 experimental samples. This means that we were possibly either oversampling the pupil or generating aliasing due to a truncation of the Zernike expansion. To study this issue experimentally, we repeated some measurements with a smaller number of samples and with different patterns. This is especially easy with LRT, which permits us high flexibility in the sampling pattern. We incremented the sampling step from 0.592 to $1 \mathrm{~mm}$, which implies recording 37 samples (rays) for a $6.5-\mathrm{mm}$ pupil. In this way we have a similar number of samples and polynomials, and thus we practically eliminate oversampling. Moreover, we compared hexagonal and square sampling patterns (both with 1-mm step size).

Figure 10 shows the results for subject RN. Each symbol represents the average of four runs, and the solid line represents the average over the three conditions: 1-mm step, square sampling (squares); 1-mm step, hexagonal (circles); 0.59-mm step, hexagonal (asterisks). Differences between conditions are lower than the error bars for most coefficients, so that we cannot see any significant difference between them. For the second subject tested, EM, the results were totally equivalent, except for slightly larger error bars (average value, $0.05 \mu \mathrm{m}$ for EM versus $0.04 \mu \mathrm{m}$ for $\mathrm{RN}$ ). This result is not surprising in normal eyes, because as Liang and Williams ${ }^{14}$ pointed out, the magnitude of aberrations decreases monotonically with the order of the Zernike polynomial (this decay is roughly exponential). For normal eyes the seventhorder coefficients are already small, so that truncation does not introduce large errors. For the two normal eyes tested, the result was basically independent of sampling pattern and step. Nevertheless, this could be different in some clinical eyes with large amounts of high-order aberrations. $^{30}$

\section{DISCUSSION AND CONCLUSIONS}

Among the different objective methods available for measuring aberrations in the human eye, we have chosen to 
compare LRT with $\mathrm{H}-\mathrm{S}$ for various reasons. (We have discarded subjective methods, as explained in Section 1). The Foucault test ${ }^{9}$ has important drawbacks, including nonlinearity, contamination of measurements by Purkinje images, and critical dependence on a perfect placement of the knife edge. Although this was one of the first objective methods that provided interesting experimental results, its use has been limited, probably as a result of to these problems. The Howland aberroscope ${ }^{7}$ has been already compared with H-S (Ref. 17); the latter provided both a finer sampling (resolution) and a higher robustness. Finally, we have discarded other methods where aberrations are not directly measured but are inferred, for instance, by means of sophisticated phase retrieval algorithms. ${ }^{13}$ On the other hand, H-S is probably becoming the most popular technique in both basic ${ }^{11,14}$ and clinical ${ }^{24}$ studies, and hence we thought that it was the best method to choose as standard reference for the comparison. Finally, we believe that LRT, having many features in common with $\mathrm{H}-\mathrm{S}$, may constitute an interesting alternative, especially in clinical applications, as we discuss below.

In summary, both methods, Laser Ray Tracing and the Hartmann-Shack wave-front sensor, have been validated for measuring ocular aberrations in a double-pass configuration. As a first step, the validation has been carried out on an artificial eye (having values of aberrations of the same order of magnitude as normal human eyes), through a direct comparison between single- and doublepass configurations, as well as by directly comparing the two methods. A further comparison between the simulated PSF's, computed from the wave-aberration data obtained with $\mathrm{H}-\mathrm{S}$ and LRT, and the single-pass PSF recorded directly on the CCD array as a primary reference permits not only a relative but also a global validation. In order for us to complete this study, the two methods (double-pass configuration) needed as well to be validated in real eyes. In this case we observed four-times-higher levels of noise, roughly the same in both the $\mathrm{H}-\mathrm{S}$ and the LRT cases, which we attribute to residual head and eye movements and, to some extent, to the low reflectivity of the retina. In fact, with real eyes we could observe some limitations of these methods. In $\mathrm{H}-\mathrm{S}$ the range of aberrations one can measure seems to be limited by the geometry of the sampling grid and the resolution of the microlenses. When either the grid or the spots are too distorted (for highly aberrated eyes) the indexing or the centroid computation, respectively, becomes difficult. This may limit the range of application of $\mathrm{H}-\mathrm{S}$, especially in some clinically interesting cases. LRT is significantly more robust because each spot is recorded and processed independently and much larger amounts of distortion (including speckle noise) can be tolerated. The main limitation in LRT comes from its sequential nature, so that it is less robust against temporal factors (eye movements, fluctuations of the accommodation, jittering of the laser scanner, etc.), and hence it is not well suited for real-time dynamic measurements. ${ }^{26}$ Interestingly, the noise level and the experimental variability in the data were equivalent in H-S and LRT (except for subject SM, because it was too difficult to estimate the centroids of several $\mathrm{H}-\mathrm{S}$ spots). This suggests that when both methods operate within their valid ranges, there seems to be a sort of ergodicity so that the different sources of experimental errors and noise (spatial and temporal) have similar effects on average, despite the different nature-parallel or sequential-of the sampling. In both cases, it was important to limit the width of the auxiliary beam (first pass in $\mathrm{H}-\mathrm{S}$ and second pass in LRT) by using artificial pupils.

Regarding other pros and cons, there are four main inherent differences between the two techniques:

1. LRT measures image (retinal or first-pass) aberrations, and $\mathrm{H}-\mathrm{S}$ measures object (aerial or second-pass) ones. However, we have obtained the same results in the two cases, which demonstrates the reversibility of light paths and the equivalence of the two types of measurements.

2. H-S uses a fixed monolithic microlens array, whereas the laser scanner can be programmed with high flexibility to change the sampling pattern in LRT. Conversely, one can change the diameter of the microlenses (by using a different array or by changing magnification), whereas it is harder to modify the width of the laser beam.

3. H-S recordings can be faster, $\sim 1 \mathrm{~s}$ to blur speckle, although other techniques could be applied to take faster real-time dynamic recordings. ${ }^{26}$ LRT measurement is limited to a few rays per second, so a fine pupil sampling may take several seconds. Jitter and eye movement can limit the accuracy of the measurement.

4. In LRT, data analysis and centroid computation are easier and more robust against noise and the presence of large aberrations, because each ray is recorded and processed independently. The sequential recording makes indexing a trivial task. In $\mathrm{H}-\mathrm{S}$ when the grid, the spots, or both are too distorted, the measurement can be difficult.

Concerning experimental difficulty, subjects' comfort, alignment and fixation, etc., we have experienced no big differences between LRT and $\mathrm{H}-\mathrm{S}$.

As a general conclusion, both methods are valid, and they are similar but have significantly different features, so one may prefer one or the other depending on the application. H-S presents clear advantages in dynamic measurements, while the present study suggests that LRT has advantages in clinical applications where one may expect to find high values of aberrations.

\section{ACKNOWLEDGEMENTS}

We thank Susana Marcos for acting as a subject and making interesting suggestions. We also thank Mike Hutley for providing us with the microlens array used in the experiments. This research has been supported by the Comisión Interministerial de Ciencia y Tecnología of Spain, grant TIC98-0925-C02-01.

The authors can be reached at the address on the title page or by e-mail: e.moreno@io.cfmac.csic.es or r.navarro@io.cfmac.csic.es. 


\section{REFERENCES}

1. J. Liang, D. R. Williams, and D. Miller, "Supernormal vision and high resolution retinal imaging through adaptive optics," J. Opt. Soc. Am. A 14, 2884-2892 (1997).

2. L. Zhu, P. C. Sun, D.-U. Bartsch, W. R. Freeman, and Y. Fainman, "Adaptive control of a micromachined continuous-membrane deformable mirror for aberration compensation," Appl. Opt. 38, 168-176 (1999).

3. R. Navarro, E. Moreno-Barriuso, S. Bará, and T. Mancebo, "Phase plates for wave-aberration compensation in the human eye," Opt. Lett. 25, 236-238 (2000).

4. T. Young, "On the mechanisms of the eye," Philos. Trans. R. Soc. London 19, 23-88 (1801).

5. A. Ivanoff, Les aberrations de l'oeil. Leur role dans l'accommodation (Editions de la revue d'Optique Théorique et Instrumentale, Paris, 1953).

6. M. S. Smirnov, "Measurement of the wave aberration of the human eye," Biofizika 6, 687-703 (1961); Biophysics 6, 776-795 (1962) (English translation).

7. H. Howland and B. Howland, "A subjective method for the measurement of monochromatic aberrations of the eye," J. Opt. Soc. Am. A 67, 1508-1518 (1977).

8. J. C. He, S. Marcos, R. H. Webb, and S. A. Burns, "Measurement of the wave-front aberration of the eye by a fast psychophysical procedure," J. Opt. Soc. Am. A 15, 2449-2456 (1998).

9. F. Berny, "Étude de la formation des images rétiniennes et détermination de l'aberration de sphéricité de l'oeil humain," Vision Res. 9, 977-990 (1969).

10. G. Walsh, W. N. Charman, and H. C. Howland, "Objective technique for the determination of monochromatic aberrations of the human eye," J. Opt. Soc. Am. A 1, 987-992 (1984).

11. J. Liang, B. Grimm, S. Golez, and J. Bille, "Objective measurement of wave aberrations of the human eye with the use of a Hartmann-Shack wave-front sensor," J. Opt. Soc. Am. A 11, 1949-1957 (1994).

12. R. Navarro and M. A. Losada, "Aberrations and relative efficiency of light pencils in the living human eye," Optom. Vision Sci. 74, 540-547 (1997).

13. I. Iglesias, E. Berrio, and P. Artal, "Estimates of the ocular wave aberration from pairs of double-pass retinal images," J. Opt. Soc. Am. A 15, 2466-2476 (1998).

14. J. Liang and D. R. Willians, "Aberrations and retinal image quality of the normal human eye," J. Opt. Soc. Am. A 14, 2873-2883 (1997).

15. R. Navarro, E. Moreno, and C. Dorronsoro, "Monochromatic aberrations and point-spread functions of the human eye across the visual field," J. Opt. Soc. Am. A 15, 2522-2529 (1998).

16. T. Salmon, L. Thibos, and A. Bradley, "Comparison of the eye's wave-front aberration measured psychophysically and with the Shack-Hartmann wave-front sensor," J. Opt. Soc. Am. A 15, 2457-2465 (1998).

17. L. Thibos and X. Hong, "Comparison of monochromatic aberrations of the human eye measured with the Howland crossed-cylinder aberroscope and the Shack-Hartmann aberrometer," presented at the OSA Annual Meeting, September 26-October 1, 1999, Santa Clara, Calif.

18. R. Navarro and E. Moreno-Barriuso, "Laser ray-tracing versus Hartmann-Shack sensor for measuring aberrations in the eye," presented at the OSA Annual Meeting, October 4-9, 1998, Baltimore, Md.

19. R. Navarro and E. Moreno-Barriuso, "Laser ray-tracing method for optical testing," Opt. Lett. 24, 1-3 (1999).

20. J. G. Sivak and R. O. Kreuzer, "Spherical aberration of the crystalline lens," Vision Res. 23, 59-70 (1983).

21. P. Artal, R. Navarro, S. Marcos, and D. R. Williams, "Odd aberrations and double-pass measurements of retinal image quality,” J. Opt. Soc. Am. A 12, 195-201 (1995).

22. D. Malacara, Optical Shop Testing, 2nd ed. (Wiley, New York, 1992).

23. J. M. Geary, Introduction to Wavefront Sensors (SPIE Optical Engineering Press, Bellingham, Wash., 1995).

24. M. W. Campbell, H. Haman, P. Simonet, and I. Brunette, "Dependence of optical image quality on refractive error: eyes after excimer laser photorefractive keratectomy (prk) versus controls," Invest. Ophthalmol. Visual Sci. 40 (Suppl.), 7 (1999).

25. Hopkins and M. J. Yzuel, "The computation of diffraction patterns in the presence of aberrations," Opt. Acta 17, 157182 (1970).

26. H. J. Hofer, J. Porter, and D. R. Williams, "Dynamic measurement of the wave aberration of the human eye," Invest. Ophthalmol. Visual Sci. 39 (Suppl.), 203 (1998).

27. S. Marcos, R. Navarro, and P. Artal, "Coherent imaging of the cone mosaic in the living human eye," J. Opt. Soc. Am. A 13, 897-905 (1996).

28. S. Marcos and R. Navarro, "Imaging the foveal cones in vivo through ocular speckle interferometry theory and numerical simulations," J. Opt. Soc. Am. A 13, 2329-2340 (1996).

29. D. Sliney and M. Wolbarsht, Safety with Lasers and Other Optical Sources, 1st ed. (Plenum, New York, 1980).

30. S. A. Burns, S. Marcos, J. McLellan, and R. H. Webb, "Role of sampling pattern and size on measuring aberrations of the eye," presented at the OSA Annual Meeting, September 26-October 1, 1999, Santa Clara, Calif. 